\title{
Emir Sader
}

\section{Die hegemoniale Krise und die Krise der Linken in Lateinamerika}

I.

Mit den Regierungen von Lula in Brasilien und Tabaré Vazquez in Uruguay wird auch die Linke von der hegemonialen Krise getroffen, die die lateinamerikanischen Gesellschaften seit der Erschöpfung des neoliberalen Modells erfahren. Regierungen, die im Zuge des Widerstands gegen die neoliberalen Politiken gewählt wurden, und in der Vergangenheit einen wichtigen Teil der Linken in ihren Ländern repräsentierten, setzten sich zwar gegen diejenigen Parteien durch, die die neoliberalen Politiken auf dem Weg gebracht haben. Doch haben auch sie den neoliberalen Pfad nicht wirklich verlassen. Noch ist es zu früh, um etwas über die möglichen Regierungen von Lopez Obrador in Mexiko und Evo Morales in Bolivien zu sagen. Aber auch in diesen Ländern besteht das Risiko einer Anpassung an das neoliberale Programm. Und die argentinische Regierung von Nestor Kirchner hat bislang, trotz kühner Initiativen (wie der Neuverhandlung der Auslandsschulden und des Widerstands gegen die Erhöhung der Benzinpreise) keine wirklich andere Wirtschaftspolitik implementiert.

Auch die Versuche der sozialen Bewegungen, die neoliberalen Regierungen aus der Macht zu drängen, sind Bestandteil dieser Krise. Ecuador bildet das beste Beispiel für ein Land, in dem die sozialen Bewegungen eine unpopuläre Regierung stürzen, eine neue Regierung an die Machst bringen, sich jedoch wiederholt verraten fühlen, und in die Opposition zurückkehren. Sowohl die Spaltung der indigenen Bewegungen, als auch die Differenzen innerhalb der bolivianischen Linken, verweisen auf ein Dilemma: Es herrscht Uneinigkeit über die Frage, ob man sich auf den institutionellen Weg einlässt, der soziale Bewegungen mit formalen politischen Akteuren zusammenbringt, oder ob man lieber nach Formen einer alternativen Politik außerhalb der bestehenden politischen Institutionen suchen soll. Die Strategie der Zapatisten etwa verbindet die Frage der Befreiung der indigenen Bevölkerung in Chiapas unmittelbar mit der Emanzipation der gesamten mexikanischen Unterklassen. Anerkannt wird damit die Notwendigkeit eines nationalen politischen Programms, einer Politik, die auf eine neue Ordnung abzielt, dabei aber vor allem auch die Konstruktion eines 
alternativen Modells von gesamtgesellschaftlichem Charakter beinhaltet. Es ist dies ein weiterer Versuch, das politische Vakuum zu füllen, welches die hegemoniale Krise auf dem lateinamerikanischen Kontinent produziert hat.

Der Neoliberalismus ist das hegemoniale Modell des gegenwärtigen Kapitalismus. Die Linke hat sich in den Widerstand zurückgezogen. Linke Politik bedeutet heute vor allem, den Neoliberalismus zu bekämpfen und (neben einer antiimperialistischen) eine antineoliberale Position zu beziehen. Die verschiedenen Strömungen innerhalb der Linken stehen für unterschiedliche Varianten des Antineoliberalismus - sei es in der radikalen Form des Antikapitalismus oder mit anderen Visionen einer postneoliberalen Gesellschaft. Allen Strömungen gemeinsam ist die antineoliberale Haltung. Nicht zufällig hat das Weltsozialforum den Antineoliberalismus als das einende Moment gewählt Das neoliberale Modell stärkte die Hegemonie des Finanzkapitals in praktisch allen lateinamerikanischen Gesellschaften. Es hat den Prozess der „Vermarktlichung" unserer Länder vorangetrieben, der bis in alle Poren unserer Gesellschaften einschließlich der staatlichen Institutionen hineinreicht. Die gesellschaftlichen Steuerungsfunktionen des Staates wurden geschwächt, die Erwerbslosigkeit und die Prekarisierung der Arbeitsverhältnisse und damit auch die Überausbeutung der Arbeit haben enorm zugenommen.

Die Kräfteverhältnisse zwischen den sozialen Klassen haben sich in der Vergangenheit radikal zum Vorteil des großen Kapitals geändert. Die politische Rechte konnte ihre Werte, ihre politischen Programme und ihre Strategien und Taktiken erneuern und eine gesellschaftliche Hegemonie erreichen, wie sie sie niemals zuvor gehabt hat.

Doch kann sich das Finanzkapital keine eigene soziale Basis schaffen, die zur Unterstützung und Legimitation seiner Macht notwendig wäre. Es schafft keine Arbeitsplätze, sondern tendiert im Gegenteil dazu, Arbeit zu eliminieren. Es erzeugt keine Umverteilung der Einkommen, sondern intensiviert die Konzentration des gesellschaftlichen Reichtums in den Händen weniger. Und es weitet die sozialen Rechte nicht aus, sondern schwächt sie. Die kleinen und mittleren Unternehmen fallen der verschärften Konkurrenz zum Opfer. Die großen Kapitale dagegen werden unter den gegebenen Bedingungen in den Exportsektor und in die Produktion von gehobenen Konsumgütern gedrängt. Worauf stützt sich dieses Modell? Vor allem auf die großen Kapitalfraktionen, die komplett oder teilweise am Prozess der Finanzakkumulation beteiligt sind. Zweitens auf die privilegierten Teile der Mittelschichten, die auf unterschiedlichen Ebenen mit dem großen internationalisierten Kapital verbunden sind. Drittens auf die Atomisierung der Gesellschaft und die enormen Schwierigkeiten, die dadurch für die Organisation sozialer Interessen und für die Formierung linker politischer Kräfte geschaffen werden. Zudem kann dieses Modell auf die ideologische Vorherrschaft der monopolisierten privaten Medien zählen - das Fernsehen spielt hier eine herausragende Rolle. 
Im Moment seiner Implementierung hatte dieses Modell unmittelbaren wirtschaftlichen Erfolg. Mit seiner Gegenreform der ökonomischen Deregulierung ergriff es die Initiative zur Bekämpfung der Inflation und der immensen öffentlichen Verschuldung, die die lateinamerikanischen Staaten in den Bankrott getrieben hatten. Doch wurde damit auch die Abhängigkeit von den internationalen Finanzinstitutionen verstärkt.

Der Erfolg des Neoliberalismus bestand zunächst im Erreichen einer monetären Stabilität. Unterstützung erfuhr er dabei nicht nur durch das große Kapital, die internationalen Finanz- und Handelsorganisationensowie die großen privaten Medienkonzerne. Es gelang auch, wie unten noch ausgeführt wird, Teile der traditionellen Linken zu kooptieren.

Doch nach dem geradezu euphorischen Klima Mitte der 1990er Jahre - das sozialistische Lager hatte sich aufgelöst und der kurze Aufschwung der nordamerikanischen Wirtschaft vermittelte die Illusion einer 'New Economy' - erlebte das neoliberale Modell eine Serie von Krisen: zunächst die Mexikokrise 1994, gefolgt von der brasilianischen Krise 1999 und der Krise in Argentinien im Jahr 2001. Nachdem Mexiko für die internationalen Organisationen zum 'Vorzeigebeispiel' geworden war und Argentinien das orthodoxeste neoliberale Modell auf dem gesamten Kontinent durchgesetzt hatte, stürzten die drei größten Ökonomien Lateinamerikas bei der Anwendung des Neoliberalismus in die Krise.

Mehr als zehn Regierungen wurden in den letzten Jahren in Lateinamerika und der Karibik als Folge der Ermüdung des Neoliberalismus gestürzt, und zwar in so verschiedenen Ländern wie Argentinien, Ecuador, Bolivien und Peru. Dabei handelte es sich um Regierungen, die die neoliberale Politik fortsetzten, wie auch um solche, die angekündigt hatten, mit dem neoliberalen Modell zu brechen, ihre Versprechen jedoch nicht einlösten. Beide Sorten von Regierungen verloren ihre Legitimität schnell, litten unter dem hohen Druck durch die Mobilisierungen der sozialistischen Bewegungen, und mussten schließlich zurücktreten.

Diese Krisen führten zur Erschöpfung des neoliberalen Modells, denn, wie oben bereits angemerkt, produziert die Hegemonie des Finanzkapitals keine ausreichende eigene soziale Basis, auf die sich die Legitimität des Modells gründen könnte. So hat sich in Lateinamerika eine Hegemoniekrise entwickelt, ein Streit zwischen dem Alten und dem Neuen, zwischen einem erschöpften Modell, das immer noch überlebt, und einer neuen Welt, die noch keine Formen gefunden hat, die den Neoliberalismus ersetzen könnten. Dies ist der Grund, weswegen Lateinamerika sich in wirtschaftlicher, sozialer und politischer Hinsicht zu einer der instabilsten Regionen der Welt entwickelt hat. 
II.

Die traditionelle Linke ist hinsichtlich des Neoliberalismus gespalten. Als das neoliberale Projekt in der Anfangsphase von der politischen Rechten - im Fall Pinochets gar von der extremen Rechten - vorangetrieben wurde, positionierte sich die Linke deutlich dagegen.. Als aber die europäische Sozialdemokratie ausgehend von den sozialistischen Parteien in Frankreich und Spanien - dieses Modell übernahm und den universellen Anspruch des sogenannten 'Washington Konsensus' akzeptierte, gab auch die lateinamerikanische Linke den Widerstand gegen den Neoliberalismus weitgehend auf.

Seinen Ausgangspunkt hatte dieser Anpassungsprozess bei den Regierungen von Carlos Menem (Argentinien), Carlos Andrés Perez (Venezuela) und Salinas de Gortari (Mexiko). Auch die chilenischen Sozialisten, die zusammen mit den Christdemokraten die Regierung Pinochet ablösten, behielten das neoliberale Modell bei. Die Politik der Regierung Fernando Henrique Cardoso in Brasilien vervollständigte den Anpassungsprozess. So unterwarfen sich praktisch alle Parteien des politischen Spektrums dem neoliberalen Konsens.

Doch es waren die Wahlerfolge von Lula in Brasilien und Tabaré Vazquez in Uruguay, die den Zyklus der Zustimmung komplettierten. Dieses mal handelte es sich um zwei Kräfte, die in den Jahrzehnten zuvor Protagonisten des Widerstands gegen das neoliberale Modell gewesen waren. Damit stellt Lateinamerika die Weltregion dar, in der sich das neoliberale Modell am stärksten durchsetzen und verallgemeinern konnte.

Die lateinamerikanische Linke vermochte bislang kein alternatives Projekt zum neoliberalen Modell zu entwickeln oder hatte zumindest nicht die Kraft, ein solches in die politische Praxis einzubringen. Die Ausnahmen kommen von den Regierungen in Kuba und Venezuela die allerdings erhebliche Besonderheiten aufweisen. Das kubanische Regime ist das Ergebnis eines revolutionären Prozesses, der die Basis des Kapitalismus und der imperialen Herrschaft im Land zerstörte. Die „boliviarische“ Regierung in Venezuela kann sich vor allem auf zwei besondere Faktoren stützen - ihre Erdölressourcen und die Unterstützung durch die Streitkräfte. So konnte sich der Neoliberalismus in Venezuela, trotz der (vergeblichen) Versuche der Regierungen von Carlos Andrés Perez und von Rafael Caldera, bislang nicht durchsetzen.

In der gegenwärtigen Epoche, in der die imperiale US-amerikanische Politik und das neoliberale Modell dominieren, ist die Linke mit einer Hegemonie konfrontiert, die die Macht des Geldes mit der Macht der Waffen kombiniert. Ein alternatives hegemoniales Projekt muss beiden Faktoren Rechnung tragen. So lässt sich etwa beobachten, wie die brasilianische Regierung mit ihrer Außenpolitik auf den zweiten dieser Aspekte außenpolitisch reagiert, das ökonomische Projekt des Neoliberalismus steht aber nicht zur Disposition. 
III.

Die Konversion der nationalistischen und sozialdemokratischen Kräfte zum Neoliberalismus, wie auch die immensen Probleme, denen die Regierungen der PT in Brasilien und der Frente Amplio in Uruguay gegenwärtig begegnen, lassen darauf schließen, dass es sich um ein komplexes Phänomen handelt, das sich nicht einfach auf eine Art 'Verrat' reduzieren lässt. Vielmehr muss dieses Kapitel der jüngsten Geschichte der lateinamerikanischen Linken vor dem Hintergrund des tiefgreifenden Wandels von Kräfteverhältnisse diskutiert werden, der in den vergangenen Jahrzehnten weltweit stattgefunden hat.

Der Abschluss der historischen Epoche, die mit dem Ende des Zweiten Weltkrieges begann und ein Gleichgewicht der Kräfte zwischen dem sozialistischen und dem kapitalistischen Lager hervorbrachte, hat fundamentale Bedeutung für den Wandel der nationalen und internationalen Kräfteverhältnisse. Dem Aufstieg des Faschismus in Europa, der die Linke in die Defensive gedrängt und ihre Konzentration auf den antifaschistischen Kampf erfordert hatte, folgte eine Phase der Restauration der politischen Stabilität und der internationalen Isolation der UdSSR. Obwohl der Impuls, der von der bolschewistischen Revolution einmal ausgegangen war, sich nun abschwächte, führte die neue Situation der Blockkonfrontation in vielerlei Hinsicht auch zu einer Wiederbelebung linker Kräfte.

Die Niederlage der Achsenmächte im Zweiten Weltkrieg, die Konstitution des sozialistischen Lagers in Osteuropa, der Sieg der chinesischen Revolution und der Prozess der Dekolonisierung in Asien und Afrika, konstituierten ein neues politisches Feld, auf dem sich das sozialistische und das kapitalistische Lager gegenüberstanden - und damit auch ein neues Gleichgewicht zwischen den großen Kräften. Die Konstitution des Lagers der 'Dritten Welt' begünstigte die Zukunftsperspektive für den antiimperialistischen und antikapitalistischen Kampf.

Diese Kräftekonstellation fand mit der Selbstauflösung des sozialistischen Lagers ein abruptes Ende. Sie mündete in eine unipolare, von der politischen, ideologischen und militärischen Offensive der USA geprägte Weltordnung.

Die unmittelbaren Konsequenzen, auch der Ablösung des keynesianischen durch das neoliberale Paradigma, waren eine Schwächung der kommunistischen Parteien, die Anpassung der sozialdemokratischen Parteien an neoliberale Programme und die Fragmentierung der Linken. Darüber hinaus kam es zu einer Schwächung des Staates und seiner steuerungs- und entwicklungspolitischen Instrumente. Die Ausdehnung der sozialen Rechte für große Teile der Bevölkerung fand ihr Ende.

Diese Umbrüche hatten auch Konsequenzen für das ideologische Profil der Linken. Mit der Blockkonfrontation zwischen dem kapitalistischen und dem sozialistischen Lager standen sich zwei unterschiedliche Interpretationen des Ost-West Konflikts gegenüber. Für das sozialistische Lager handelte es sich um 
eine Konfrontation zwischen Sozialismus und Kapitalismus, für das kapitalistische Lager ging es um Demokratie und Totalitarismus. Mit dem Sieg des kapitalistischen Blocks setzte sich auch dessen Weltsicht durch, gemäß deren es sich im 20. Jahrhundert um einen Kampf zwischen der Demokratie und dem Totalitarismus gehandelt hatte. Letzterer hatte dem zufolge zunächst die Form des Nationalsozialismus und des Faschismus angenommen, anschließend trat er in Gestalt des Kommunismus auf (der Nationalismus und der Islamismus galten als Varianten des Totalitarismus). Ebenfalls siegte die Gleichsetzung der Demokratie mit dem Liberalismus, was für die politische und ideologische Hegemonie der liberalen Ideologie einen fundamentalen Aspekt darstellt.

Ein weiteres wichtiges Element der der ideologischen Hegemonie des Neoliberalismus besteht darin, das Scheitern des staatszentrierten sowjetischen Modells mit der Finanzkrise des Wohlfahrtsstaates zu assoziieren, und dadurch das Konzept des intervenierenden Staates generell zu disqualifizieren. Statt dessen setzen die neoliberalen Ideologen auf die Kräfte des Marktes. In ihrem Diskurs polarisieren sie die Begriffe staatlich und privat und verbinden dies mit einer Gegenüberstellung von Staat und Zivilgesellschaft

Die lateinamerikanische Linke konnte sich diesen Einflüssen nicht entziehen. Sie übernahm die Gleichsetzung der Demokratie mit der liberalen Demokratie. Sie akzeptierte die Interpretation, dass die Finanzkrise des Staates ein Anzeichen der definitiven Erschöpfung des Staates in seiner Rolle als politischer, ökonomischer und sozialer Agent sei, und sie ging dazu über, statt dessen die Zivilgesellschaft als Akteur stark zu machen - mit all ihren Konnotationen von 'Citizenship' bis 'Netzwerkgesellschaft'. Dies ist die gegenwärtig 'gültige Vision'. Sie geht mit der These vom Verlust der Zentralität der Arbeit einher und ersetzt die Vorstellung von Klassenwidersprüchen durch Theorien der sozialen Exklusion - mit einer klaren funktionalistischen Verkürzung.

Die neue Qualität der Regierungen von Lula und Tabaré Vazquez besteht in der ausdrücklichen Akzeptanz des Liberalismus durch linke Kräfte. Die brasilianische Regierung hielt anfänglich an der von Cardoso geerbten Politik fest, um so einer möglichen ökonomischen Destabilisierung vorzubeugen und die Phase des Übergangs hin zu einer neuen Regierung und einer neuen Politik zu erleichtern. Doch schnell wurde aus dieser vorläufigen Strategie ein permanentes Programm, einschließlich einer Haushaltspolitik, die noch härter als die der vorherigen Regierung ist. Auch die Regierung von Tabaré in Uruguay übernahm das geerbte Modell, das die Politik der Haushaltskonsolidierung und der monetären Stabilität gegenüber sozialpolitischen Zielen klar bevorzugt. Die Fortführung des neoliberalen Modells bedeutet nicht nur soziale Politik faktisch einzuschränken sondern auch, dass statt einer Ausweitung sozialer und politischer Rechte eine auf 'Problemgruppen' fokussierte Politik und eine Politik der Notversorgung betrieben wird. 


\section{IV.}

Nachdem sich die Linke gegenüber dem Neoliberalismus gespalten hat, leisten Teile der Linken aus der Opposition heraus Widerstand, während andere das neoliberale Modell in der Praxis umsetzen. Wenn letztere, wie im Falle Brasiliens und Uruguays, an die Regierung gelangen und das herrschende ökonomische Regime fortsetzen, wird das Ausmaß der Krise, in der sich die lateinamerikanische Linke befindet, deutlich.

Zunächst einmal handelt sich um eine Krise des ideologischen Selbstverständnisses, weil die linken Parteien, die an die Regierung gekommen sind, weder mit dem neoliberalen Modell noch mit ihren eigenen Werten gebrochen haben. Es mangelt ihnen an ideologischer Stärke, um eine alternative politische Praxis zu initiieren, und Werte zu vertreten, die sich gegen das neoliberale Ideal wenden. Mit Sicherheit hat der Einfluss des Liberalismus zur Schwächung der Linken beigetragen und sie dazu gebracht, antikapitalistische Positionen und die Fragen nach Arbeitsverhältnissen, nach Klassenwidersprüchen, nach dem Prozess der Kapitalakkumulation und nach Bündnispartnern für den politischen Widerstand aufzugeben. Auch steht die Linke unter dem Einfluss der These von der irreversiblen Krise des Staates und der Notwendigkeit fiskalpolitischer Maßnahmen zum Erhalt der Währungsstabilität - nur so ließe sich ein angeblich 'nachhaltiges Wachstum' wiedererlangen.

Die großen privaten Medienkonzerne stützen diesen ideologischen Konsens. Die Diskussion um Steuerreformen, die auf Umverteilung abzielen und öffentliche Investitionen finanzieren könnten, wird nicht geführt. Die Priorität monetärer Stabilitätspolitik wird angesichts der öffentlichen Schulden als unabweisbar behauptet.

Vorstellungen von der Ausweitung sozialer Rechte und von demokratischen Reformen, die auf eine Stärkung der öffentlichen Sphäre abzielen, sind großen Teilen der Linken weitgehend abhanden gekommen. Angesichts der Tatsache, dass die neoliberale Ideologie auf eine Polarisierung der Kategorien staatlich und privat abzielt, gilt es für die Linke, die soziale Realität statt dessen entlang der Begriffe öffentlich versus marktförmig zu thematisieren. Dies ist die Aufgabe, der sich eine postneoliberale Bewegung stellen muss.

Bislang hat die traditionelle Linken es nicht geschafft, aus dem neoliberalen Modell, das keines der großen gesellschaftlichen Probleme des Kontinents lösen oder auch nur mildern konnte, auszubrechen. Deshalb müsste es vor allem um die Stärkung des Antineoliberalismus gehen. Mit der Fortsetzung des Neoliberalismus verwüsten Regierungen und Parteien ursprünglich linker Herkunft jedoch das originär linke politische Feld.

Es lassen sich also eine Reihe von Merkmalen benennen, die die Krise der lateinamerikanischen Linken charakterisieren - und die möglicherweise auch auf andere Regionen der Welt zutreffen: 
- Die faktische Abwesenheit politischer Kräfte, die in der Lage wären, sich der Konstruktion eines antineoliberalen Projekts anzunehmen und einen Block antineoliberaler Kräfte zu bilden.

- Die Schwächung der traditionellen sozialen Basis der Linken - Gewerkschaftsbewegungen, Arbeiter und Angestellte des öffentlichen Sektors, Intellektuelle der öffentlichen Sphäre - und die Fragmentierung und Atomisierung der Arbeitswelt.

- Die Hegemonie liberaler Ideen und Konzepte auf dem Feld der Linken selbst. Dies drückt sich in der mechanischen Identifikation der Demokratie mit der liberalen Demokratie aus, in der Abwesenheit jeder antikapitalistischen Kritik, in der Akzeptanz gegenüber Marktmodellen, in der Ablösung universeller und inklusiver Politik durch eine Politik jeweils einzelner Notprogramme und des staatlichen Rückzugs als Konsequenz der Stabilitätspolitik.

Die Konstruktion eines hegemonialen postneoliberalen Projekts bedarf vor allem einer Analyse der jüngsten gesellschaftlichen Transformationsprozesse unter den Bedingungen neoliberaler Politik:

- Dazu muss eine neue 'Geographie der Arbeit' entwickelt werden, samt einer Auseinandersetzung mit den multiplen informellen Räumen der Gesellschaft. Ohne eine solche Debatte wird das Thema der Arbeit - das weiterhin strategischen Charakter hat - auf die Dimensionen formaler Arbeitsbeziehungen und klassischer Gewerkschaftspolitik beschränkt bleiben. Ohne diese Auseinandersetzung hat eine Überwindung der Isolation der Linken und der sozialen Bewegungen keine Perspektive, vor allem im Hinblick auf die neue Generation der Prekarisierten.

- Es gilt, die historische Epoche der neoliberalen Hegemonie - mit all ihren Stärken und Schwächen - grundlegend zu verstehen. Dazu muss vor allem die überragende Fähigkeit des Neoliberalismus zur Herstellung ideologischer Hegemonie untersuchen werden, seine Fähigkeit, ausgehend vom 'american way of life', der heute die Gedanken vieler Menschen in praktisch allen Ländern der Welt beherrscht, Ideale und Normen zu beeinflussen.

- Schließlich müsste es gelingen, eine soziale, politische, ideologische und organisatorische Kraft zu erlangen, die es erlaubt, eine postneoliberale Alternative zu formulieren und zu konstruieren.

Die Liste der Anforderungen ließe sich noch verlängern. Sollen die Anforderungen auch erfüllt werden, sind vor allem zwei fundamentale Prinzipien zu beachten: 1) 'Ohne revolutionäre Theorie keine revolutionäre Praxis' und 2) 
'In Klassengesellschaften, ist die Ideologie der herrschenden Klasse die herrschende Ideologie'. Es handelt sich insofern um 'Prinzipien', als sie tief in der Realität verankert sind. Auch wenn man sie manchmal gern vergisst, begegnen wir ihnen immer wieder als konstitutiv im Kampf gegen die kapitalistischen Gesellschaften, als unabkömmliche Orientierungspunkte jeder sozialen und politischen Praxis, die sich der Transformation der Realität annimmt. Das erste Prinzip weist darauf hin, dass die politische Praxis jeden Mangel an theoretischer Durchdringung der Verhältnisses unerbittlich bestraft, dass es ohne eine Dechiffrierung der komplizierten gesellschaftlichen Wirklichkeit nicht möglich ist, die Theorie als ein Instrument zur gesellschaftlichen Transformation zu nutzen. Dies gilt umso mehr angesichts des starken institutionellen und medialen Drucks, über den sich die Hegemonie der liberalen Ideen durchsetzt und verankert. Ohne theoretische Stärke und ohne programmatische, strategische und taktische Vorschläge ist die Kooptation der linken Kräfte unter diesen Bedingungen geradezu unvermeidbar.

Das zweite Prinzip verweist auf die Notwendigkeit, ein alternatives Projekte zu formulieren, um der gegenwärtigen Tendenz der Anpassung an die existierende Politik, an die existierenden Institutionen und an den durch die großen privaten Medienkonzerne fabrizierten Konsens nicht noch Vorschub zu leisten. Es gilt, ein Verständnis für die Stärke der liberalen Hegemonie auf ökonomischer, politischer und sozialer Ebene und im Hinblick auf die allgegenwärtige Verbreitung der Kultur des 'american way of life' zu entwickeln.

Im Kern handelt es sich also darum, die Herausforderung, die vor uns liegt, in all ihren Dimensionen anzuerkennen: ein soziales, politisches, kulturelles und moralisches postneoliberales Projekt zu formulieren, und die Kraft zu erlangen, es in die Wirklichkeit umzusetzen.

Übersetzung aus dem Spanischen: Henrik Lebuhn 